\title{
Asymmetric Nickel-Catalyzed Intramolecular Hydroalkenylation
}

\section{Gategory}

Metal-Catalyzed

Asymmetric

Synthesis and

Stereoselective

Reactions

\section{Key words}

nickel catalysis

hydroalkenylation

piperidines

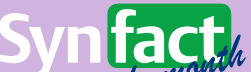
of the manth
Significance: Nickel catalysis has been explored in recent years as a cost-effective alternative to palladium. Zhu, Zhou, and co-workers report the enantioselective nickel-catalyzed isomerizations of dienes to yield chiral piperidines or tetrahydropyrans, which are found in a number of natural products and drug structures.<smiles>[Z1]C1C[Y]CC(=C)C1[Tl]</smiles>

28 examples $22-98 \%$ yield $51-99 \%$ ee

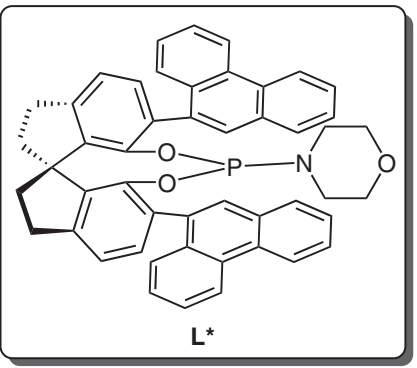

Selected examples:<smiles>C=C1C[N]CCC1c1ccccc1</smiles>

$69 \%$ yield, $99 \%$ ee<smiles>C=C1CN([As])C[C@H](C)C1c1ccccc1</smiles>

$69 \%$ yield, $99 \%$ ee<smiles>C=C1CN([Al])CCC1c1ccc(Br)cc1</smiles>

$88 \%$ yield, $99 \%$ ee<smiles>C=C1CN(C)CC(c2ccccc2)[C@H]1c1ccccc1</smiles>

$69 \%$ yield, $99 \%$ ee<smiles>C=C1CN([Al-])CCC1c1ccc(C(C)=O)cc1</smiles>

$88 \%$ yield, $98 \%$ ee<smiles>C=C1CN([Al])CCC1c1cccs1</smiles>

$98 \%$ yield, $99 \%$ ee

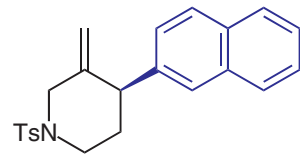

$88 \%$ yield, $98 \%$ ee

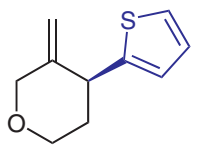

$63 \%$ yield, $99 \%$ ee

Example applied in the enantioselective synthesis of (+)-femoxetin:<smiles>C=C1C[N]CCC1c1ccccc1</smiles>

$\frac{\text { 1. } \mathrm{NBS}, \mathrm{Bu}_{3} \mathrm{P}(61 \% \text { yield })}{\text { 2. } n-\mathrm{Bu}_{3} \mathrm{SnH}, \mathrm{Et}_{3} \mathrm{~B}, 91 \% \text { yield }}$<smiles>OC[C@@H]1C[14CH2]CC[C@@H]1c1ccccc1</smiles><smiles>COc1ccc(OC[C@H]2CN(C)CCC2c2ccccc2)cc1</smiles>

SYNFACTS Contributors: Mark Lautens, Andrew Whyte

Synfacts 2018, 14(08), 0821 Published online: 18.07.2018 DOI: 10.1055/s-0037-1610488; Reg-No.: L07718SF

Comment: The reaction was proposed to involve a nickel-hydride intermediate, which was generated through $\beta$-hydride elimination of the alkyl nickel species. The approach was successful with a variety of substituents on the olefin, and all the reactions proceeded with high enantioselectivities. 